УДК 616.711-073.432.1

DOI: $10.26435 /$ UC.V0I1(30).272

\author{
А.Д. Зубов ${ }^{1}$, А.А. Бережная ${ }^{2}$, Л.Н. Антонова ${ }^{1}$ \\ ${ }^{1}$ ГОО ВПО «Донецкий национальный медицинский университет имени М. Горького», Донецк \\ ${ }^{2}$ Донецкое клиническое территориальное медицинское объединение, Донецк
}

\title{
РОЛЬ УЛЬТРАЗВУКОВОГО ИССЛЕДОВАНИЯ В КОМПЛЕКСНОЙ ДИАГНОСТИКЕ ШЕЙНОЙ РАДИКУЛОПАТИИ (ОБЗОР ЛИТЕРАТУРЫ)
}

Заболевания позвоночника относятся к наиболее распространенным, приводящим к значительным материальным и трудовым потерям [1, 2]. Наиболее частой причиной боли в спине является радикулопатия (корешковый синдром) - патологическое состояние периферической нервной системы, развивающееся вследствие непосредственного механического воздействия (компрессии) на корешки спинномозговых нервов и асептического воспаления в корешковой зоне и периневральных структурах [3-7]. Причиной радикулопатии выступают остеохондроз, спондилез, протрузии и грыжи межпозвонковых дисков, сужение фораминального канала, унковертебральный артроз. Ведущими патогенетическими факторами вертеброгенных радикулопатий являются компрессионные и рефлекторные механизмы, воспалительные и аутоиммунные процессы, микроциркуляторные расстройства в области поражения [8, 9].

Шейная радикулопатия является распространенным патологическим состоянием: заболеваемость в популяции составляет 107 на 100000 мужчин и 63 на 100000 женщин в год [5, 7]. Эпизод боли в шее в течение жизни как минимум однократно отмечался у двух третей взрослого населения [4]. При вертеброгенной шейной радикулопатии корешковая боль сопровождается клиническими признаками дисфункции корешка в виде нарушений чувствительности (ощущение онемения, парестезии, дизестезии, аллодиния, гиперпатия, гипералгезия) в соответствующем дерматоме, выпадения глубоких рефлексов, мышечной слабости в дистальных или проксимальных отделах верхних конечностей. Цервикалгия требует нозологической идентификации в каждом конкретном клиническом случае для обеспечения индивидуализированного выбора тактики лечения пациента [10].

Выраженность болевогосиндрома можетбыть оценена количественно, для чего применяют- ся визуально-аналоговые шкалы (10-балльная и 100-балльная), шкалы вербальных оценок, опросники Мак-Гилла, HAQ, WOMAC и др. Высказывается мнение об отсутствии прямого влияния степени дегенеративно-дистрофических изменений позвоночника на выраженность болевого синдрома $[11,12]$.

Отношение к традиционному клиническому обследованию, включая и неврологический статус, среди специалистов неоднозначно. По мнению ряда авторов, его результаты недостаточны для определения локализации и выраженности поражений структур позвоночного столба [4]. Другие исследователи указывают, напротив, что в подавляющем большинстве случаев острой и хронической боли в шее бывает достаточно клинического обследования, а необходимости в дополнительных инструментальных исследованиях нет $[13,14]$. Указывается, что их целесообразно проводить только при подозрении на вертеброгенную патологию (спондилолистез, перелом). Методом выбора в таких ситуациях до настоящего времени остается рентгенография в двух основных проекциях и с функциональными нагрузками в положении максимального сгибания и разгибания. МРТ и рентгеновская компьютерная томография рекомендуются при выявлении т.н. «красных флажков» (наличие симптомов или факторов риска тяжелого заболевания, травмы, метастазов, инфекционного процесса и пр.), а также при решении вопроса о необходимости хирургического лечения $[13,14]$. Проведение рентгенографии, МРТ и СКТ показано только пациентам с болью в шее III и IV градаций по Neck Pain Task Force, 2008 [15].

Рентгенография в прямой и боковой проекциях до настоящего времени остается основным методом визуализации заболеваний по-

(c) А.Д. Зубов, А.А. Бережная, Л.Н. Антонова, 2019 (c) Университетская Клиника, 2019 
звоночника на этапе скрининга. В литературе приведены показания и особенности выполнения различных спондилограмм (прямые, боковые, косые, функциональные, через открытый рот) [16].

Основной целью рентгенографии в прямой и боковой проекциях является исключение таких причин боли, как опухоль, спондилит или остеопороз.

При рентгенографии шейного отдела могут быть выявлены такие проявления дегенеративно-дистрофических изменений, как: снижение высоты диска, субхондральный остеосклероз, формирование остефитов, артроз межпозвонковых суставов, изменение формы позвонков, смещения позвонков, искривления оси позвоночника $[17,18]$, а также склероз замыкательных пластин, гипертрофию суставных отростков, неравномерное сужение межпозвонковой щели. Описаны технологии оценки и измерения структур шейного отдела по прямым и боковым рентгенограммам, основные морфологические признаки и критерии стадии развития остеохондроза $[16,19]$. Рентгенография с функциональными пробами (функциональная рентгенография) используется для определения нестабильности отдельных сегментов позвоночника и скрытых спондилистезов [15].

Компьютерная томография (КТ) имеет ряд существенных преимуществ, к которым относится: возможность определения соотношения и топографии анатомических и патологических структур, получение изображения всего поперечного среза области и одномоментная сравнительная оценка изображения симметричных отделов тела, определение общей формы и характера поверхности исследуемого объекта, возможность выполнения денситометрии и пр. Спиральная КТ (СКТ) высокоинформативна в выявлении дистрофических изменений шейного отдела позвоночника, превосходит МРТ в оценке изменений костных структур, в том числе проявлений артроза межпозвонковых суставов, обызвествлений фиброзного кольца диска и желтой связки [20]. СКТ может быть успешно использована с целью определения размеров позвоночного канала, площади межпозвонкового отверстия, объема межпозвонкового канала, угла дугоотростчатого сустава по отношению к фронтальной оси тела позвонка, площади поперечного сечения отверстия позвоночной артерии и пр. Она хорошо выявляет стеноз межпозвонкового отверстия, остеофиты, оссификацию задней продольной связки [15]. Указывается на повышение информативности СКТ посредством проведения ротационных проб для определения объема движений позвоночника при осевом вращении - т.н. функциональная компьютерная томография [21].

Однако СКТ, как и рентгенография, имеет значимые ограничения в визуализации мягкотканных структур, недостаточно информативна для оценки состояния связочного аппарата и корешков спинномозговых нервов [16]. Недостатками метода также являются значительная лучевая нагрузка, высокая себестоимость, длительность процедуры и необходимость значительное время соблюдать неподвижность, что затруднительно при выраженном болевом синдроме, статичность получаемого изображения, меньшая визуальная дифференцированность различных сред, возможность индуцированной клаустрофобии [20].

Магнитно-резонансная томография (МРT) лучше позволяет выявлять и оценивать состояние межпозвонкового диска и спинного мозга, оценить структуру и степень дегидратации пульпозного ядра межпозвонкового диска, выявлять дегенеративные процессы в межпозвонковых дисках, разрывы и трещины фиброзного кольца, смещения пульпозного ядра при протрузиях диска, грыжи дисков размером 2 мм, оценивать степень отека и венозного стаза в эпидуральной и окружающей клетчатке, степень компрессии корешков и дурального мешка [22]. В диагностике грыж межпозвонковых дисков чувствительность СКТ и МРТ составляет соответственно 93,7\% и 98,9\%, специфичность - 82,6\% и 96,8\%, точность - 89,1\% и 97,8\% [16]. Также МРТ предоставляет возможность визуализации вторичных изменений в телах позвонков и дугоотростчатых суставах, вызывающих компрессионный синдром [23]. Метод МРТ целесообразно использовать для выявления изменений межпозвонковых дисков, определения степени компрессии дурального мешка, спинного мозга, оценки выраженности миелопатии, определения размеров межпозвонковых отверстий. МРТ шейного отдела показана при планировании оперативного вмешательства по поводу грыжи диска, опухоли шейной локализации спинного мозга, а также при подозрении на инфекционно-воспалительное и метастатическое (вторичное) поражение позвоночника.

Ряд авторов сообщает, что при МРТ достаточно хорошо визуализируются нервы большого диаметра, окруженные жировой прослойкой, однако нервы малого диаметра практически не дифференцируются от других тканей [24]. Несмотря на высокую контрастность мягких тканей и возможность оценки объекта в разных плоскостях, МРТ периферических нервов остаётся менее предпочтительным методом диагностики по сравнению с УЗИ [24]. 
Недостатками метода являются, прежде всего, его ограниченная доступность и высокая стоимость оборудования и наивысшая среди всех современных инструментальных диагностических методов стоимость самого обследования [6, 16], длительность выполнения. Ограничения метода вызваны и техническими особенностями выполнения обследования -необходимость сохранять неподвижность продолжительное время при наличии болевого синдрома [6], невозможность проведения при наличии металлических объектов в организме (протезов, фиксирующих металлоконструкций стимуляторов, инородных тел и пр.). Также, сообщается, что МРТ, выполняемая в положении пациента лежа, недоцценивает сагиттальное смещение при дегенеративном спондилолистезе по сравнению с вертикальной боковой рентгенограммой [25]. Ряд авторов указывает на возможность проведения т.н. кинематической МРТ в положениях сгибания и разгибания, которая позволяет выявить нестабильность шейного отдела позвоночника [16].

Авторы указывают, что в течение еще значительного периода времени МРТ не сможет стать методом выбора тактики и контроля эффективности терапии [1], что обусловлено ограниченным числом установок, длительностью и высокой стоимостью исследования [1]. В связи с этим в отечественной лучевой диагностике заболеваний позвоночника важная роль отводится доступным методам. В связи с возможностью оценки изменений мягкотканых компонентов наиболее информативным методом первичной диагностики признается УЗИ [6].

При интерпретации данных МРТ, как и других лучевых методов визуализации (КТ, рентгенография), важно учитывать, что выявление рентгенологических признаков дегенеративнодистрофических изменений позвоночника чаще всего не имеет клинического значения, так как их можно обнаружить у подавляющего большинства лиц зрелого и пожилого возраста [17]. Сообщается, что дегенеративно-дистрофические изменения, протрузии и грыжи дисков в отсутствие каких-либо симптомов цервикалгии отмечаются у 60\% пациентов после 40 лет и более чем у 80\% после 60 лет [13]. Рентгенографические признаки остеохондроза и спондилоартроза позвоночника обнаруживаются почти у половины лиц среднего возраста и у большинства пожилых людей [6]. При этом сообщается, что выраженность этих изменений не коррелирует с клинической картиной и не исключает других причин болей в спине, в частности, протрузий диска, межпозвонковых грыж, сужения спинно-мозгового канала, эпидурита, ли- гаментита желтой связки) [13]. По другим данным, у 57\% обследованных, не имевших жалоб на боль в шейном отделе, обнаруживаются протрузии дисков, у 26\% - стеноз позвоночного канала, у 7\% - грыжи дисков с признаками сдавления спинного мозга [6]. Кроме того, сообщается, что выявляемые при МРТ изменения в зоне компрессии не всегда соответствуют неврологической симптоматике [16].

Данные электронейромиографии редко имеют практическую значимость при вертеброгенной радикулопатии, но иногда важны в дифференциальной диагностике с поражением периферического нерва или сплетения. Дифференцирующим признаком является скорость проведения возбуждения по двигательным волокнам, которая у пациентов с радикулопатией обычно остается нормальной даже при выявлении слабости в пораженном миотоме, поскольку только часть волокон в пределах нерва оказывается поврежденной [26].

Такие инвазивные методики, как миелография, дискография, эпидурография, в настоящее время применяются ограничено, по строгим показаниям, в основном в рамках предоперационной диагностики $[6,17]$.

Традиционно среди специалистов сложилось предубеждение о малой информативности ультразвукового исследования (УЗИ) в диагностике патологии опорно-двигательного аппарата, в частности, позвоночника, в связи с высокой отражающей способностью костной ткани [1].

В 90-е годы в литературе появились первые сообщения о трансабдоминальном УЗИ поясничного отдела позвоночника. Японские авторы в исследовании на трупах подтвердили, что высота межпозвонкового диска может быть определена с помощью УЗИ, пульпозное ядро в норме является гипоэхогенным, а фиброзное кольцо - гиперэхогенным [27]. Первые сообщения о роли УЗИ в диагностике патологии межпозвонковых дисков и позвоночного канала (на поясничном уровне) в отечественной литературе были представлены в 1992 г. [28].

В настоящее время большинством авторов признается, что современные ультразвуковые технологии, оптимально подобранные датчики и существующие методики проведения исследования позволяют получать изображение хрящевых структур межпозвонковых дисков [1] и оценивать изменения в паравертебральных тканях [29]. УЗИ межпозвонковых дисков шейного отдела, в отличие от КТ и рентгенографии, полностью исключает лучевую нагрузку на пациента; метод неинвазивен, прост в применении, не требует специальных помещений, больших затрат времени и средств [30]. Достоинствами ме- 
тода также являются: непосредственный контакт с пациентом во время обследования, что дает возможность детализации жалоб и субъективного восприятия своего состояния больным; возможность ультразвуковой пальпации исследуемой области и определение болезненности конкретной зоны при этом, возможность повторных исследований за короткие промежутки времени [29]. Также к преимуществам УЗИ могут быть отнесены возможность применения динамических тестов в процессе исследования без контрастирования; более четкая дифференцируемость жидкостных структур, в т.ч. сосудов, допплерографическая оценка кровотока.

Метод, однако, имеет и ряд недостатков, к которым относят: большую роль субъективного фактора в проведении исследования и интерпретации полученных данных; ограниченную возможность стандартизации исследования в аспектах доступов, положения датчиков; наличие непреодолимых акустических сред, прежде всего, костных структур [1].

В литературе имеется ряд публикаций, описывающих возможности УЗИ в исследовании позвоночника, описана нормальная ультразвуковая картина вертебральных структур, исследованы корреляции между ультразвуковым изображением межпозвонковых дисков и их анатомическими и макроморфологическими параметрами как в норме, так и при наличии патологических изменений $[6,10]$.

Подавляющее большинство авторов признают, что УЗИ является простым способом визуализации, который позволяет дифференцировать нормальный и дистрофически измененный межпозвонковый диск [1, 31]. Также проведено сопоставление результатов УЗИ с миелографией, КТ, МРТ и дискографией [6].

Показаниями к ультразвуковому исследованию шейного отдела позвоночника и выходящих нервных стволов считают: корешковые компрессионные синдромы как проявления вертеброгенной шейной радикулопатии, которые обусловлены медианными и боковыми грыжами, протрузиями межпозвонковых дисков и характеризуются выпадением чувствительной и двигательной функций в зоне иннервации периферических нервов шейного сплетения; болевые синдромы, обусловленные шейным остеохондрозом или наличием иной патологии (протрузии дисков, листез тел позвонков, остеофиты, спондилез, спондилоартроз); планируемое ортопедическое лечение (с целью выявления требуемой степени декомпрессии), клинические признаки компрессии позвоночной артерии в костном канале для определения ее возможных вертеброгенных причин [1].
УЗИ межпозвонковых дисков и позвоночного канала до настоящего времени осуществляется по Л.Г. Плеханову с соавт [28] с изменениями и дополнениями, внесенными А.Ю. Киндзерским [1]. Как правило, для оценки состояния позвоночника используется двухмерное УЗИ, при необходимости может быть выполнено исследование в режимах 3D и 4D с получением объемного изображения пораженной области. При сагиттальном сканировании определяют форму, взаиморасположение, размеры, структуру переднего контура тел позвонков и межпозвонковых дисков. При сканировании в горизонтальной анатомической плоскости оценивают особенности структуры межпозвонковых дисков и спинномозгового канала.

При УЗИ позвоночника фиксируют ряд параметров и проводят стандартные измерения. Эхографически в сагиттальном сечении оценивают толщину межпозвонкового диска, в аксиальном - структуру и форму пульпозного ядра, толщину заднего сегмента фиброзного кольца, наличие участков истончений, разволокнений, разрывов, размеры участков протрузий фиброзного кольца и грыжевого выпячивания [32].

Методика проведения УЗИ шейного отдела позвоночника до настоящего времени несовершенна и может быть оптимизирована за счет стандартизации исследования путем определения четких анатомических ориентиров, стандартных проекций и режимов сканирования, а также определения качественных и количественных нормативных параметров межпозвонковых дисков, позвоночного канала, корешковых каналов [31].

При УЗИ неизмененного шейного отдела позвоночника определяются гиперэхогенные контуры позвонков с четкой акустической тенью от тел и частично поперечных отростков; межпозвонковые диски визуализируются в виде гипоэхогенных структур, за которыми идут гиперэхогенные линейные сигналы от твердой и мягкой мозговых оболочек, между которыми находится анэхогенное ликворное пространство, а также содержимое позвоночного канала с единичными мелколинейными включениями. Передний контур дисков в норме не выступает за пределы тел позвонков [1].

Передние поверхности тел позвонков в норме при исследовании в сагиттальной плоскости определяются как гиперэхогенные горизонтальные линии, за которыми находится акустическая тень [132]. В норме крючковидные отростки шейных позвонков не контактируют с телом вышележащего позвонка; при развитии патологического процесса, сопровождающегося уплощением и нарушением структуры межпозвон- 
кового диска, указанные отростки могут образовывать с телами позвонков патологические унковертебральные сочленения [18].

Между телами позвонков визуализируются межпозвонковые диски, состоящие из пульпозного ядра, лишенного кровоснабжения и иннервации, фиброзного кольца и располагающихся по его передней и задней поверхностям соответственно передней и задней продольных связок [18]. Высота межпозвонкового диска оценивается как расстояние между наиболее сближенными поверхностями тел позвонков, прилежащими к диску [6, 23]. Дорсальнее межпозвонкового диска четко визуализируется овальный анэхогенный спинномозговой канал, ограниченный гиперэхогенным контуром - эпидуральным жиром. Дорсальнее задней стенки спинномозгового канала определяется гиперэхогенная зона, представляющая собой отраженный от дужек позвонков ультразвуковой сигнал [13].

Немногочисленные исследования посвящены роли УЗИ в диагностике патологии позвоночника. В литературе описаны эхопризнаки различных дегенеративно-дистрофических изменений позвоночника, в первую очередь, различных проявлений остеохондроза, наиболее изученным является поясничный отдел [1]. Сообщается о выявлении протрузий и грыж в поясничном отделе [30]. Указывается о возможности УЗ дифференцировки протрузии (выпячивания диска в позвоночный канал за счет пролабирования фиброзного кольца без его разрыва и вовлечения в патологический процесс эпидуральной клетчатки и корешков спинномозговых нервов) и грыжи разрыва волокон фиброзного кольца с выходом фрагмента пульпозного ядра через образовавшееся отверстие в позвоночный канал) [1].

Сообщений о возможности исследования шейного отдела значительно меньше в связи с существенными ограничениями его визуализации. Указывается, что при ультразвуковом исследовании в режиме реального времени с функциональной пробой (сгибание-разгибание) могут быть выявлены нестабильность шейного отдела позвоночника и нарушение соотношения позвонков [6]. Эхографически возможно определить спондилолистез [40], выявить патологию межпозвонкового диска, возникшую вследствие его обезвоживания, дегомогенизации и нарушения структуры: снижение высоты диска, уменьшение размеров пульпозного ядра, повышение его эхогенности, а в дальнейшем и изменение его структуры в виде фрагментации и появления гиперэхогенных включений [4].

В сагиттальной плоскости возможно выявление протрузии межпозвонкового диска. Она эхографически визуализируется как выбухание заднего контура межпозвонкового диска по дуге большого радиуса без признаков разрыва фиброзного кольца, но с признаками рубцовых изменений в прилежащей эпидуральной клетчатке в виде гиперэхогенных линейных включений [29]. Грыжи межпозвонковых дисков морфологически представляют собой участки выпадения пульпозного ядра сквозь разрывы в фиброзном кольце и эхографически определяются как выбухания заднего контура межпозвонкового диска по дуге малого радиуса с разрывом фиброзного кольца [33]. Ультразвуковыми признаками грыжи межпозвонковых дисков также являются: уменьшение размеров спинномозгового канала по сравнению с соседними межпозвонковыми дисками (за счет рубцовых изменений, локализованных по заднему контуру фиброзного кольца), уменьшение размера корешкового канала по сравнению с контралатеральным [1].

Наибольшую чувствительность и специфичность УЗИ имеет при выявлении патологических изменений передней поверхности позвоночника - протрузии межпозвонковых дисков (соответственно 88,6\% и 98,3\% [33], размеров спинномозгового канала (соответственно 90,9\% и 100,0\%), остеофитов [30]. Для патологии внутренних структур и заднего контура межпозвонковых дисков (грыжи дисков, сужение корешковых каналов) диагностическая ценность УЗИ несколько ниже [23] (исследования проводились для поясничного отдела позвоночника).

Также в литературе представлены сведения о возможностях ультразвуковой визуализации шейного отдела у детей, в частности, с цервикальным болевым синдромом, с целью выявления анатомических особенностей, приобретенных деформаций и органических изменений костных и мягкотканных структур [34].

По мнению ряда авторов, УЗИ в сравнении с рентгенологическим методом является более информативным для оценки мягких тканей позвоночного столба, поскольку позволяет оценить структуру межпозвонковых дисков и спинномозгового канала, и по диагностической ценности приближается к МРТ [6].

Допплерографические исследования при шейной радикулопатии до настоящего времени ограничиваются оценкой кровотока в позвоночных артериях [16].

Роль УЗИ в диагностике корешкового синдрома до настоящего времени не раскрыта. Метод не включен в стандарты обследования больных с корешковым синдромом [31].

Первое сообщение о возможности УЗ визуализации периферических нервов было сделано B. Fornage [35] в 1988 г. Автором описана уль- 
тразвуковая картина нервного ствола и приемы его идентификации относительно анатомических ориентиров. Однако низкая на тот момент разрешающая способность ультразвуковой аппаратуры не позволила достоверно распознать контуры таких тонких структур, как нервные сплетения [36]. Первые сообщения о возможностях ультразвуковой визуализации и оценки состояния нервных сплетений и периферических нервов конечностей стали появляться только к концу девяностых годов прошлого столетия [36].

В настоящее время УЗИ является активно развивающимся методом исследования периферических нервов. Указывается, что контрастность изображений и уровень пространственного разрешения, получаемые при УЗИ, значительно выше, чем при МРТ [26]. Осевое разрешение современных высокочастотных УЗ датчиков 12-17 МГц достигает 250-500 мкм, что позволяет с максимальной из применяемых в настоящее время методов визуализации точностью дифференцировать нервный ствол и оценить взаимоотношение с окружающими тканями [37]. К преимуществам метода УЗИ следует также отнести возможность оценки васкуляризации и гемодинамики в зоне интереса.

Однако большинство исследований нервных структур посвящены диагностике заболеваний и повреждений периферических нервов, а также повреждениям шейного и плечевого сплетений у новорожденных [36, 38]. Вопросы визуализации шейного и плечевого сплетений у взрослых затрагивают преимущественно работы, посвященные региональной анестезии [39]. Ультразвуковые исследования спинномозговых нервов шейного отдела отображены в единичных сообщениях - публикации R. Beekman и coавт. [40] и значительной по объему и подробной работе Е.Г. Салтыковой [36], которая, к сожалению, не охватывает вопросы шейной радикулопатии.

При исследовании шейного отдела определяются тела и частично боковые отростки позвонков; из позвоночного ствола отходят гипоэхогенные тяжи - передние ветви спинномозговых нервов, которые при УЗИ визуализируются как гипоэхогенные тяжи, выходящие из позвоночного ствола, делающие угловой изгиб сразу при выходе из канала, идущие в дистальном направлении межмышечно и плотно соприкасающиеся друг с другом [36]. При сканировании в поперечной плоскости нервные стволы определяются как гипоэхогенные овальные структуры, окруженные соединительной тканью и жировой клетчаткой, имеющей неоднородную структуру, умеренно пониженную эхогенность с хаотично расположенными тонкими гиперэхогеными линиями $[36,40]$.

Для визуализации доступны исходящие из позвоночного столба спинномозговые нервы на уровне $\mathrm{C}_{\mathrm{II}}-\mathrm{C}_{\mathrm{VII}}$. Сообщается, что качество визуализации зависит от телосложения пациента. Так, у лиц астенического телосложения, с длинной шеей удается визуализировать нервы на уровне $\mathrm{C}_{\mathrm{II}}-\mathrm{C}_{\mathrm{VII}}$, нормостенического телосложения - $\mathrm{C}_{\mathrm{III}}-\mathrm{C}_{\mathrm{VII}}$, У лиц гиперстенического телосложения с короткой шеей и выраженным подкожножировым слоем исследование затруднительно и, как правило, ограничено визуализацией нервов $\mathrm{C}_{\mathrm{IV}}-\mathrm{C}_{\mathrm{VI}}$ [36]. Корешок $\mathrm{C}_{\mathrm{I}}$ визуализировать при УЗИ не представляется возможным вследствие высокого анатомического расположения и малых размеров нерва. При сканировании в поперечной плоскости визуализация верхних шейных корешков на уровне $\mathrm{C}_{\mathrm{II}}-\mathrm{C}_{\mathrm{III}}$ затруднена у 42,5\% из-за трудностей, связанных с дифференциацией тканей [36].

Ряд исследователей указывает, что для объективного ультразвукового исследования нервов в диагностике необходимо выделить количественные характеристики, отражающие морфологические изменения нерва, подлежащие математико-статистическому анализу [27, 41, 42], однако до настоящего времени этот вопрос остается открытым.

В литературе имеется ряд указаний на возможность УЗ оценки диаметра периферических нервов [4, 43 и др.], поперечных размеров: толщины, ширины и площади поперечного сечения [43]. Большинство авторов высоко оценивают возможности УЗИ в оценке размеров периферических нервов. Так, Э. Ю. Малецкий с соавт. [43] и Moayeri N. [44] на основе сопоставления УЗ измерений периферических нервов с результатами МРТ и стандартного интраоперационного измерения нерва указывают на высокие коэффициенты линейной и ранговой корреляции и делают заключение о высоком уровне воспроизводимости при соблюдении общепринятой методики УЗ измерения нерва. Исследователи указывают, что количественные результаты, полученные при УЗИ, стабильно менее реальных анатомических размеров, и объясняют этот факт особенностями ультразвуковой визуализации нерва и методикой его измерения, а именно исключением наружного гиперэхогенного ободка (наружного эпиневрия) и измереним только отчетливо дифференцируемого гипоэхогенного тяжа [43].

Указывается (для периферических нервов), что эхографически определяемая площадь поперечного сечения нерва является информативным диагностическим параметром [41] прово- 
дят сопоставление полученных данных со стандартными антропометрическими параметрами - рост, масса тела, индекс массы тела. Также предложено оценивать значение интраневральной вариабельности - отношение максимальной и минимальной площади сечения [41, 45], и определять градиент асимметрии как отношение средних площадей сечения нерва справа и слева, уплощения нерва как соотношение ширины нерва к его толщине [43]. Другие авторы [4] сообщают, что УЗ оценка утолщения нервного ствола имеет высокий уровень погрешности и не может быть использована как информативный диагностический критерий. В отношении спинномозговых нервов такие исследования не проводились.

Анализ литературных данных показывает, что шейная радикулопатия является широко распространенным во всем мире заболеванием. Она характеризуется тенденцией к хронизации и прогрессированию процесса, потерей трудоспособности и инвалидизацией. Кроме того, в последние годы наблюдается рост заболеваемости у молодых и трудоспособных людей. Это обуславливает необходимость своевременной диагностики, лечения и профилактики остеохондроза, и как его следствия - шейной радикулопатии.

Вместе с тем существующие на сегодня методы лучевой визуализации шейного отдела позвоночника, а именно рентгенография, КТ, МРТ, которые являются золотым стандартом диагностики шейной радикулопатии, помимо своих преимуществ имеют ряд недостатков. Рентгенография и КТ не предоставляют информации о состоянии мягких тканей, и связаны с лучевой нагрузкой, выполнение МРТ ограничено высокой стоимостью, а также наличием металличе- ских имплантов и протезов, что делает актуальной проблему поиска альтернативных методов диагностики.

В настоящее время изучаются новые возможности широко распространенного метода УЗИ для диагностики шейной радикулопатии. Это исследование не оказывает лучевого воздействия на пациента, поэтому его можно выполнять многократно без ограничения временных интервалов между осмотрами, не нанесет вреда беременным женщинам, онкологическим пациентам, а также лицам с ослабленным иммунитетом.

Ультразвуковая визуализация предоставляет возможность выявления и оценки патологических изменений костных и мягкотканных структур шейного отдела позвоночника. Однако в неврологической практике возможности УЗИ еще не до конца раскрыты, в научной литературе имеются лишь единичные упоминания о возможностях метода, а в повседневной клинической практике он в указанных целях не используется. Несмотря на наличие единичных публикаций, недостаточно освещены вопросы нормальной ультразвуковой анатомии шейного отдела позвоночника, не изучены изменения, развивающиеся у больных шейной радикулопатией. Предоставляемая УЗИ возможность исследования мягкотканых структур, в частности, нервных стволов, до настоящего времени не нашла практического использования в клинической неврологии.

Таким образом, ультразвуковая диагностика является перспективным методом обследования пациентов с шейной радикулопатией, однако ее возможности на сегодняшний день недостаточно изучены, что указывает на важность дальнейших исследований в данном направлении.

\section{А.Д. Зубов" ${ }^{1}$ А.А. Бережная ${ }^{2}$, Л.Н. Антонова ${ }^{1}$}

${ }^{1}$ ГОО ВПО «Донецикий национальный медицинский университет им. М. Горького», Донецик

${ }^{2}$ Донецкое клиническое территориальное медицинское объединение, Донецк

\section{РОЛЬ УЛЬТРАЗВУКОВОГО ИССЛЕДОВАНИЯ}

\section{В КОМПЛЕКСНОЙ ДИАГНОСТИКЕ ШЕЙНОЙ РАДИКУЛОПАТИИ (ОБЗОР ЛИТЕРАТУРЫ)}

Изучено современное состояние вопроса диагностики шейной радикулопатии - широко распространенного заболевания, имеющего тенденцию к хронизации, потере трудоспособности и инвалидизации. Традиционные методы лучевой визуализации шейного отдела позвоночника - рентгенография, рентгеновская компьютерная и магнитно-резонансная томография - наряду с достоинствами имеют ряд недостатков: рентгенологическая визуализация не предоставляет информации о состоянии мягких тканей и связана с лучевой нагрузкой, доступность магнитно- резонансной томографии ограничена, что делает актуальной проблему поиска альтернативных методов диагностики.

Достоинством ультразвукового исследования является доступность, быстрота, полипозиционность, отсутствие лучевой нагрузки, возможность многократного выполнения. Однако в диагностике шейной радикулопатии его возможности еще не до конца раскрыты, в научной литературе имеются лишь единичные упоминания о возможностях метода, а в повседневной клинической практике он в указанных целях 
не используется. Имеющиеся в литературе данные об исследовании других отделов позвоночника и периферических нервов, а также единичные сообщения об ультразвуковых исследованиях вертебральных и мягкотканных структур шейного отдела позволяют прогнозировать высокую эффективность ультразвуковой визуализации в диагностике шейной радикулопатии и перспективность дальнейших исследований данной проблемы.

Ключевые слова: шейная радикулопатия, лучевая диагностика, ультразвуковое исследование.

\section{A.D. Zubov ${ }^{1}$, A.A. Berezhnaya ${ }^{2}$, L.N. Antonova ${ }^{1}$}

${ }^{1}$ SEI HPE «M. Gorky Donetsk National Medical University», Donetsk ${ }^{2}$ Donetsk Clinical Territorial Medical Association, Donetsk

\section{ROLE OF ULTRASOUND IN THE COMPLEX DIAGNOSIS OF CERVICAL RADICULOPATHY (LITERATURE REVIEW)}

The current state of the issue of the diagnosis of cervical radiculopathy - widespread disease with a tendency to chronicity, incapacitation and disability, has been studied. Traditional methods of radiation imaging of the cervical spine - radiography, X-ray computed and magnetic resonance imaging - along with the advantages have several disadvantages: X-ray visualization does not provide information about the state of soft tissues and is associated with radiation exposure, the availability of magnetic resonance imaging is limited, which makes the problem of finding alternative diagnostic methods relevant.

The advantage of ultrasound is the availability, speed, polypositionality, the absence of radiation exposure, the possibility of multiple performance. However, in the di- agnosis of cervical radiculopathy, its capabilities are not yet fully disclosed, there are only a few references in the scientific literature about the possibilities of the method, and in everyday clinical practice it is not used for these purposes. Available in the literature data on the study of other parts of the spine and peripheral nerves, as well as isolated reports of ultrasound studies of the vertebral and soft tissue structures of the cervical section, allow us to predict the high efficiency of ultrasound imaging in the diagnosis of cervical radiculopathy and the promising outlook of further research on this problem.

Key words: cervical radiculopathy, radiodiagnosis, ultrasound.

\section{ЛИТЕРАТУРА}

1. Кинзерский А.Ю. Ультразвуковая диагностика остеохондроза позвоночника. Киев: ВБО «Украинский доплеровский клуб», 2007. 136.

2. Голубева В. Л. (ред.). Болевые синдромы в неврологической практике. М.: МЕДпресс-информ; 2010. 330.

3. Смирнова О.И. Эффективность применения вертебральной терапии в комплексном лечении дорсопатий шейного отдела позвоночника. Инновационная наука. 2015; 3: 236-239.

4. Мументалер М., Штер М., Мюллер-Фаль Г. Поражения периферических нервов и корешковые синдромы. Москва: МИА, 2014. 616.

5. Ходос Х.-Б.Г. Нервные болезни. Руководство для врачей. 5-е изд. Москва: МИА, 2013. 616.

6. Laker S.R., Concannon L.G. Radiologic Evaluation of the Neck: A Review of Radiography, Ultrasonography, Computed Tomography, Magnetic Resonance Imaging, and Other Imaging Modalities for Neck Pain. Physical Medicine and Rehabilitation Clinics of North America. 2011; 4: 17-22. doi:10.1016/j.pmr.2011.03.010

7. Tarulli A. Neurology. A clinician's approach. Cambridge: University press, 2011.225.

8. Kakitsubata Y., Theodorou S.J., Theodorou D.J., Nabeshima K., Kakitsubata S., Tamura S. Sonographic Characterization of the Lumbar Interverebral Disk With Anatomic Correlation and Histopathologic Findings. J. Ultrasound Med. 2005; Vol. 24, 4: 489-499. doi:10.1002/jum.14894

9. Kokubo Y., Uchida K. , Kobayashi S. , Yayama T., Sato R., Nakajima H., Takamura T., Mwaka E.S. , Orwotho N. T, Bangirana A., Baba H. Herniated and spondylotic intervertebral discs of the human cervical spine: histological and immunohistological findings in 500 en bloc surgical samples. Laboratory investigation. J Neurosurg. 2008; 9: 285295. doi:10.3171/SPI/2008/9/9/285

10. Шостак Н.А., Правдюк Н.Г. Боль в шее: дифференци-

\section{REFERENCES}

1. Kinzerskij A.Ju. Ultrasound diagnosis of spinal osteochondrosis. Kiev: VBO «Ukrainskij doplerovskij klub», 2007. 136. (in Russian).

2. Golubeva V. L. (eds.). Pain syndromes in neurological practice. Moscow: MEDpress-inform; 2010. 330. (in Russian).

3. Smirnova O.I. Effectiveness of using vertebral therapy in the complex treatment of dorsopathies of the cervical spine. Innovacionnaya nauka. 2015; 3: 236-239. (in Russian).

4. Mumentaler M., Shter M., Mjuller-Fal' G. Peripheral nerve lesions and radicular syndromes. Moskva: MIA, 2014. 616. (in Russian).

5. Hodos H.-B.G. Nervous diseases. Guide for doctors. 5-e izd. Moscow: MIA, 2013. 616. (in Russian).

6. Laker S.R., Concannon L.G. Radiologic Evaluation of the Neck: A Review of Radiography, Ultrasonography, Computed Tomography, Magnetic Resonance Imaging, and Other Imaging Modalities for Neck Pain. Physical Medicine and Rehabilitation Clinics of North America. 2011; 4: 17-22.

7. Tarulli A. Neurology. A clinician's approach. Cambridge: University press, 2011.225.

8. Kakitsubata Y., Theodorou S.J., Theodorou D.J., Nabeshima K., Kakitsubata S., Tamura S. Sonographic Characterization of the Lumbar Interverebral Disk With Anatomic Correlation and Histopathologic Findings. J. Ultrasound Med. 2005; Vol. 24, 4:489-499. doi:10.1002/jum.14894

9. Kokubo Y., Uchida K. , Kobayashi S. , Yayama T., Sato R., Nakajima H., Takamura T., Mwaka E.S., Orwotho N. T, Bangirana A., Baba H. Herniated and spondylotic intervertebral discs of the human cervical spine: histological and immunohistological findings in 500 en bloc surgical samples. Laboratory investigation. J Neurosurg. 2008; 9: 285295. doi:10.3171/SPI/2008/9/9/285

10. Shostak N.A., Pravdjuk N.G. Neck pain: differential di- 
альный диагноз и основные подходы к лечению. Лечебное дело. 2009; 2: 54-59.

11. Товажнянская Е.Л. Цервикалгии: рациональный выбор противоболевой терапии. Международный неврологический журнал. 2014; 2 (64): 123-129.

12. Чернышева Т.В. Багирова Г.Г. Валидация русскоязычных версий некоторых опросников у больных с синдромом боли в нижней части спины. Научно-практическая ревматология. 2005; 4: 24-33.

13. Чечет Е.А., Парфенов В.А. Ведение пациентов с болью в шее. Неврология, нейропсихиатрия, психосоматика. 2016; 8 (1): 4-8.

14. Cohen S.P. Epidemiology, diagnosis, and treatment of neck pain. Mayo. Clin. Proc. 2015; 90 (2): 284-299. doi:10.1016/j.mayocp.2014.09.00

15. Исайкин А.И. Боль в шее: причины, диагностика, лечение. Неврология, нейропсихиатрия, психосоматика. 2011; 4: 94-98.

16. Захматова Т.В., Щедренок В.В., Могучая О.В. Контентанализ информации о клинико-лучевой диагностике повреждений и дегенеративных заболеваний шейного отдела позвоночника (обзор литературы). Вестник Российского научного центра рентгенорадиологии Минздрава России. 2016; 2: 20-44.

17. Левин О.С., Макотрова Т.А. Вертеброгенная шейная радикулопатия. РМЖ - Русский медицинский журнал. 2012; 12: 21-28.

18. Чехонацкий И.А., Чехонацкий В.А. Лучевая диагностика шейного отдела позвоночника при краниоцервикальной травме в остром периоде. Саратовский научно-медицинский журнал. 2016; 12 (2): 222-223.

19. Михайлов А.Н. Лучевая визуализация дегенеративнодистрофических заболеваний позвоночника и суставов. Минск: БелМАПО, 2015. 155.

20. Park M.S., Lee Y.B., Moon S.H., Lee H.-M., Kim T.-H., Oh J. B., Riew K. D. Facet joint degeneration of the cervical spine: A computed tomographic analysis of 320 patients. Spine. 2014; 39 (12): e713-e718. doi:10.1097/ BRS.0000000000000326

21. Игнатьев Ю.Т., Болотов Д.А., Власова Г.А., Смяловский В.Э., Рождественский А.С., Черненко М.С. Функциональная компьютерная томография с оценкой ротационной подвижности шейного отдела позвоночника у больных с цервикогенной головной болью. Мануальный терапевт. 2010; 40 (4): 44-50.

22. Олизарович М.В., Олизарович Е.М. Секвестрация многоуровневых грыж поясничных межпозвонковых дисков. Проблемы здоровья и экологии. 2016; 2: 42-48.

23. Салина Е.А., Лихачева Е.Б., Лутошкина Е.Б., Шоломов И.И., Любицкий И.И., Чехонацкий А.А. Клиниконейровизуализационные особенности вертеброгенной радикулопатии в сочетании с гемангиомами позвонков. Саратовский научно-медицинский журнал. 2012; 8 (2): 521-524.

24. Айтемиров Ш.М., Нинель В.Г., Коршунова Г.А., Щаницын И.Н. Высокоразрешающая ультрасонография в диагностике и хирургии периферических нервов конечностей. Травматология и ортопедия России. 2015; 3 (77): 116-125.

25. Chaput C.D., Allred J.J., Pandorf J.J., Song J., Rahm M.D. The significance of facet joint cross-sectional area on magnetic resonance imaging in relationship to cervical degenerative spondylolisthesis. The Spine J. 2013; V. 13, 8: 856861. doi:10.1016/j.spinee.2013.01.021

26. Бабанов С.А., Бараева Р.А. Болевые феномены в шейном отделе позвоночника: оптимизация диагностических и лечебных мероприятий. Consilium Medicum. 2014; 2: 115-121.

27. Kakitsubata Y., Nabeshima K., Theodorou D.J. Lumbar intervertebral disc: ultrasonography with anatomic correlation in cadavers. Radiology. 1999; 213 (1) (Suppl.): 544.

28. Плеханов Л.Г., Жовтановский О.М., Кинзерский А.Ю., Полляк Л.Н. Способ ультразвукового исследования межпозвонковых дисков и позвоночного канала на поясничном уровне. В кн.: Патология позвоночника. СПб, 1992: 123-126. agnosis and main treatment approaches. Lechebnoe delo. 2009; 2: 54-59. (in Russian).

11. Tovazhnjanskaja E.L. Cervicalgia: a rational choice of pain therapy. Mezhdunarodnyj nevrologicheskij zhurnal. 2014; 2 (64): 123-129. (in Russian).

12. Chernysheva T.V., Bagirova G.G. Validation of the Russian versions of some questionnaires in patients with lower back pain. Nauchno-prakticheskaja revmatologija. 2005; 4: 24-33. (in Russian).

13. Chechet E.A., Parfenov V.A. Maintaining patients with neck pain. Nevrologija, nejropsihiatrija, psihosomatika. 2016; 8 (1): 4-8. (in Russian).

14. Cohen S.P. Epidemiology, diagnosis, and treatment of neck pain. Mayo. Clin. Proc. 2015; 90 (2): 284-299. doi:10.1016/j.mayocp.2014.09.00

15. Isajkin A.I. Neck pain: causes, diagnosis, treatment. Nevrologija, nejropsihiatrija, psihosomatika. 2011; 4: 94-98. (in Russian).

16. Zahmatova T.V., Shhedrenok V.V., Moguchaja O.V. Content Analysis Information about Clinical and Beam Diagnostics of Injuries and Degenerative Diseases of the Cervical Spine (a Review of the Literature). Vestnik Rossijskogo nauchnogo centra rentgenoradiologii Minzdrava Rossii. 2016; 2: 20-44. (in Russian).

17. Levin O.S., Makotrova T.A. Vertebral cervical radiculopathy. RMZh - Russkij medicinskij zhurnal. 2012; 12:21-28. (in Russian).

18. Chehonackij I.A., Chehonackij V.A. Radiation diagnosis of the cervical spine in craniocervical trauma in the acute period. Saratovskij nauchno-medicinskij zhurnal. 2016; 12 (2): C. 222-223. (in Russian).

19. Mihajlov A.N. Radiographic visualization of degenerative-dystrophic diseases of the spine and joints. Minsk: BelMAPO, 2015. 155. (in Russian).

20. Park M.S., Lee Y.B., Moon S.H., Lee H.-M., Kim T.-H., Oh J. B., Riew K. D. Facet joint degeneration of the cervical spine: A computed tomographic analysis of 320 patients. Spine. 2014; 39 (12): e713-e718. doi:10.1097/ BRS.0000000000000326

21. Ignat'ev Ju.T., Bolotov D.A., Vlasova G.A., Smjalovskij V.Je., Rozhdestvenskij A.S., Chernenko M.S. Functional computed tomography with assessment of the rotational mobility of the cervical spine in patients with cerviogenic headache. Manual'nyj terapevt. 2010; 40 (4): 44-50.

22. Olizarovich M.V., Olizarovich E.M. Sequestration of multilevel hernias of lumbar intervertebral discs. Problemy zdorov'ja i jekologii. 2016; 2: 42-48. (in Russian).

23. Salina E.A., Lihacheva E.B., Lutoshkina E.B., Sholomov I.I., Ljubickij I.I., Chehonackij A.A. Clinical and neuroimaging features of vertebral radiculopathy combined with vertebral hemangiomas. Saratovskij nauchno-medicinskij zhurnal. 2012; 8 (2): 521-524. (in Russian).

24. Ajtemirov Sh.M., Ninel' V.G., Korshunova G.A., Shhanicyn I.N. High-resolution ultrasonography in the diagnosis and surgery of peripheral nerves of the limbs. Travmatologija i ortopedija Rossii. 2015; 3 (77): 116-125. (in Russian).

25. Chaput C.D., Allred J.J., Pandorf J.J., Song J., Rahm M.D. The significance of facet joint cross-sectional area on magnetic resonance imaging in relationship to cervical degenerative spondylolisthesis. The Spine J. 2013; V. 13, 8: 856861. doi:10.1016/j.spinee.2013.01.021

26. Babanov S.A., Baraeva R.A. Pain phenomena in the cervical spine: optimization of diagnostic and therapeutic measures. Consilium Medicum. 2014; 2: 115-121. (in Russian).

27. Kakitsubata Y., Nabeshima K., Theodorou D.J. Lumbar intervertebral disc: ultrasonography with anatomic correlation in cadavers. Radiology. 1999; 213 (1) (Suppl.): 544.

28. Plehanov L.G., Zhovtanovskij O.M., Kinzerskij A.Ju., Polljak L.N. Ultrasound method of intervertebral discs and spinal canal at the lumbar level. V kn.: Patologija pozvonochnika. SPb, 1992: 123-126. (in Russian).

29. Shutikhina I.V., Tsybulskaya Y.A., Smerdin S.V., Selyukova N.V., Baturin O.V., Kokov L.S. Capabilities of combined application of multislice linear digital x-ray tomography and ultrasound examination in diagnosing spinal tuber- 
29. Шутихина И.В, Цыбульская Ю.А., Смердин С. В, Селюкова Н.В., Батурин О.В., Коков Л.С. Возможности совместного использования многосрезовой линейной цифровой рентгеновской томографии и ультразвукового исследования в диагностике туберкулезного поражения позвоночника. Современные технологии в медицине. 2015; 8 (4): 82-91. doi:10.17691/stm2016.8.4.11

30. Тухбатуллин М.Г., Алиева И.М. Современные ультразвуковые технологии в клинической практике. Практическая медицина. 2012; 5 (60): 30-35.

31. Пономаренко С.А. Трансабдоминальная ультрасонография поясничного отдела позвоночника: методология, нормальная анатомия. Світ медицини та біології. 2015; 2 (49): 60-65.

32. Vergari C., Rouch P., Dubois G., Bonneau D. Non-invasive biomechanical characterization of intervertebral discs by shear wave ultrasound elastography: a feasibility study. Eur. Radiol. 2014; V. 24, 12: 3210-3216. doi:10.1007/ s00330-014-3382-8

33. Федянин С.А., Шумахер Г.И., Федянин А.С., Маликов А.С., Осинцева Л.В., Дорохова Е.А. Возможности ультразвукового исследования в диагностике причин рецидива болевого синдрома в отдаленном периоде оперативного лечения грыж межпозвонковых дисков. Бюллетень сибирской медицины. 2008; 5: 198-200.

34. Бахтеева Н.Х., Ионова Т.А., Белоногов В.Н., Бажанов С.П., Островский В.В. Патологические изменения в шейном отделе позвоночника у детей с цервикальным болевым синдромом. Ортопедия, травматология и восстановительная хирургия детского возраста. 2016; 4 (4): 12-20. doi:10.17816/PTORS4412-20

35. Fornage B. D. Peripheral nerves of the extremities: imaging with US. Radiology. 1988; 167 (1): 179-182.

36. Салтыкова В.Г. Митьков В.В. Методика ультразвукового исследования шейного и плечевого сплетений. Ультразвуковая и функциональная диагностика. 2008; 6: 76-87.

37. Мартель И.И., Мещерягина И.А., Митина Ю.Л., Россик О.С., Михайлова Е. А. МРТ-диагностика повреждений периферических нервов. Бюллетень ВСНЦ СО РАМН. $2011 ; 4$ (80), Ч. 1: 119-123.

38. Noto Y. Nerve ultrasound is useful for the diagnosis of neuromuscular diseases. Rinsho Shinkeigaku. 2013; 23 (11): 1215-1216. doi:10.5692/clinicalneurol.53.1215

39. Herring A.A. The ultrasound-guided superficial cervical plexus block for anesthesia and analgesia in emergency care settings. Am. J. Emerg. Med. 2012; 30 (7): 1263-1267. doi:10.1016/j.ajem.2011.06.023

40. Beekman R., van den Berg L.H., Franssen H., Visser L.H., van Asseldonk J.T.H., Wokke J.H.J. Ultrasonography shows extensive nerve enlargements in multifocal motor neuropathy. Neurology. 2005; 65 (2): 305-307. doi:10.1212/01. wnl.0000169179.67764.30

41. Наумова Е.С., Дружинин Д.С., Никитин С.С., Курбатов С.А. Спектр сонографических изменений при наследственной моторно-сенсорной нейропатии с аутосомно-доминантным и Х-сцепленным наследованием. Нервно-мышечные болезни. 2016; 6 (6): 27-34.

42. Гажонова В.Е., Абельцев В.П., Емельяненко М.В., Онищенко М.П. Технологии Fusion при исследовании мышечно-скелетной системы. Медицинская визуализация. 2016; 4: 109-118.

43. Малецкий Э.Ю., Короткевич М.М., Бутова А.В., Александров Н.Ю., Ицкович И.Э. Измерение периферических нервов: сопоставление ультразвуковых, магнитно-резонансных и интраоперационных данных. Медицинская визуализация. 2015; (2): 78-86.

44. Moayeri N., van GeffenG.J., Bruhn J., Chan V.W., Groen G.J. Correlation among ultrasound, cross-sectional anatomy, and histology of the sciatic nerve: a review. Reg. Anesth. Pain. Med. 2010; 35 (5): 442-449.

45. Дружинин Д.С., Наумова Е.С. Никитин С.С. Ультразвуковая визуализация периферических нервов при мультифокальной моторной нейропатии и хронической воспалительной демиелинизирующей полинейропатии. Нервно-мышечные болезни. 2016; 6 (6): 63-73. culous lesion. Sovremennye tehnologii v medicine 2016; 8 (4): 82-91. (in Russian).. doi:10.17691/stm2016.8.4.11

30. Tuhbatullin M.G., Alieva I.M. Modern ultrasound technology in clinical practice. Prakticheskaja medicina. 2012; 5 (60): 30-35. (in Russian).

31. Ponomarenko S.A. Transabdominal ultrasonography of the lumbar spine: methodology, normal anatomy. Svit medicini ta biologii. 2015; 2 (49): 60-65. (in Russian).

32. Vergari C., Rouch P., Dubois G., Bonneau D. Non-invasive biomechanical characterization of intervertebral discs by shear wave ultrasound elastography: a feasibility study. Eur. Radiol. 2014; V. 24, 12: 3210-3216. doi:10.1007/ s00330-014-3382-8

33. Fedyanin S.A., Shumakher G.I., Fedyanin A.S., Malikov A.S., Osintseva L.V., Dorokhova Ye.A. Potentialities of the ultrasonic examination by detection of the pain syndrome relapse causes in the late period of the disk herniation surgical treatment. Bjulleten' sibirskoj mediciny. 2008; 5: 198-200. (in Russian).

34. Bakhteeva N.Kh., Ionova T.A., Belonogov V.N., Bazhanov S.P., Ostrovskij V.V. Pathological changes of the cervical spine in children with cervical pain syndrome. Ortopedija, travmatologija i vosstanovitel'naja hirurgija detskogo vozrasta. 2016; 4 (4): 12-20. (in Russian).. doi:10.17816/ PTORS4412-20

35. Fornage B. D. Peripheral nerves of the extremities: imaging with US. Radiology. 1988; 167 (1): 179-182.

36. Saltykova V.G., Mitkov V.V. Methodology of Ultrasound Investigation of the Cervical and Brachial Plexuses. Ul'trazvukovaja i funkcional'naja diagnostika. 2008; 6: 7687. (in Russian).

37. Martel I.I., Meshcheryagina I.A., Mitina Yu.L., Rossik O.S., Mikhailova E.A. MR-image diagnostics of injuries of peripheral nerves. Bjulleten' VSNC SO RAMN. 2011; 4 (80), part 1: 119-123. (in Russian).

38. Noto Y. Nerve ultrasound is useful for the diagnosis of neuromuscular diseases. Rinsho Shinkeigaku. 2013; 23 (11): 1215-1216. doi:10.5692/clinicalneurol.53.1215

39. Herring A.A. The ultrasound-guided superficial cervical plexus block for anesthesia and analgesia in emergency care settings. Am. J. Emerg. Med. 2012; 30 (7): 1263-1267. doi:10.1016/j.ajem.2011.06.023

40. Beekman R., van den Berg L.H., Franssen H., Visser L.H., van Asseldonk J.T.H., Wokke J.H.J. Ultrasonography shows extensive nerve enlargements in multifocal motor neuropathy. Neurology. 2005; 65 (2): 305-307. doi:10.1212/01. wnl.0000169179.67764.30

41. Naumova E.S., Druzhinin D.S., Nikitin S.S., Kurbatov S.A. The spectrum of sonographic changes in hereditary motor-sensory neuropathy with autosomal dominant and Xlinked inheritance. Nervno-myshechnye bolezni. 2016; 6 (6): 27-34. (in Russian).

42. Gazhonova V.Y., Abelcev V.P., Emelianenko M.V., Onischenko M.P. Fusion Navigation in Evaluation of MusculoSkeletal System. Medical Visualization. 2016; (4): 109-118. (in Russian)

43. Maletskiy E.Y., Korotkevich M.M., Butova A.V., Alexandrov N.Y., Itskovich I.E. Measurements of Peripheral Nerves: Comparison of Ultrasound, MRI and Direct Intraoperative Data. Medical Visualization. 2015; (2): 78-86. (in Russian).

44. Moayeri N., van Geffen G.J., Bruhn J., Chan V.W., Groen G.J. Correlation among ultrasound, cross-sectional anatomy, and histology of the sciatic nerve: a review. Reg. Anesth. Pain. Med. 2010; 35 (5): 442-449.

45. Druzhinin D.S., Naumova E.S., Nikitin S.S. Ultrasound imaging of peripheral nerves in multifocal motor neuropathy and chronic inflammatory demyelinating polyneuropathy. Nervno-myshechnye bolezni. 2016; 6 (6): 63-73. (in Russian). 